Research Article

\title{
Hidden Geometry of Bidirectional Grid-Constrained Stochastic Processes
}

\author{
Aldo Taranto $(1)$ and Shahjahan Khan \\ School of Sciences, University of Southern Queensland Toowoomba, Toowoomba, QLD 4350, Australia \\ Correspondence should be addressed to Aldo Taranto; aldo.taranto@usq.edu.au
}

Received 24 March 2021; Revised 18 April 2021; Accepted 4 May 2021; Published 25 May 2021

Academic Editor: Marek T. Malinowski

Copyright (c) 2021 Aldo Taranto and Shahjahan Khan. This is an open access article distributed under the Creative Commons Attribution License, which permits unrestricted use, distribution, and reproduction in any medium, provided the original work is properly cited.

Bidirectional Grid Constrained (BGC) stochastic processes (BGCSPs) are constrained Itô diffusions with the property that the further they drift away from the origin, the more the resistance to movement in that direction they undergo. The underlying characteristics of the BGC parameter $\Psi\left(X_{t}, t\right)$ are investigated by examining its geometric properties. The most appropriate convex form for $\Psi$, that is, the parabolic cylinder is identified after extensive simulation of various possible forms. The formula for the resulting hidden reflective barrier(s) is determined by comparing it with the simpler Ornstein-Uhlenbeck process (OUP). Applications of BGCSP arise when a series of semipermeable barriers are present, such as regulating interest rates and chemical reactions under concentration gradients, which gives rise to two hidden reflective barriers.

\section{Introduction}

In the work of Taranto et al. [1], the concept of Bidirectional Grid Constrained (BGC) stochastic processes (BGCSPs) was described as a general Itô diffusion, in which the further it drifts away from the origin, the more constrained the Ito diffusion(s) becomes. In this paper, we extend that research by geometrically examining various potential forms of $\Psi\left(X_{t}, t\right)$ and showing that the parabolic cylinder is the ideal form for BGCSP. All other researches on BGCSPs examine the application of BGSCPs to algorithmic trading, so this paper extends the probability theory aspects of BGCSP. We note that, for an arbitrary stochastic function $h$, the following notations are equivalent:

$$
h(X(t))=h(X, t)=h\left(X_{t}\right)=h\left(X_{t}, t\right),
$$

and the last of these is adopted here. We will also interchange between

$$
\Psi\left(X_{t}, t\right)=\Psi(x, t)=\Psi(x),
$$

depending on the specific context. The stochastic differential equation (SDE) of BGC stochastic processes was defined as follows.
Definition 1 (Definition I of BGC Stochastic Processes). For a complete filtered probability space $\left(\Omega, \mathscr{F},\{\mathscr{F}\}_{t \geq 0}, \mathbb{P}\right)$ and a BGC function $\Psi(x): \mathbb{R} \longrightarrow \mathbb{R}, \forall x \in \mathbb{R}$, the corresponding BGC Itô diffusion is expressed as

$$
\mathrm{d} X_{t}=(f\left(X_{t}, t\right) \underbrace{-\operatorname{sgn}\left[X_{t}\right] \Psi\left(X_{t}, t\right)}_{\mathrm{BGC}}) \mathrm{d} t+g\left(X_{t}, t\right) \mathrm{d} W_{t},
$$

where $\operatorname{sgn}[x]$ is the sign function defined in the usual sense, $f\left(X_{t}, t\right)$ is the drift term, $\Psi\left(X_{t}, t\right)$ is the constraining term, $g\left(X_{t}, t\right)$ is the diffusion term, and $f\left(X_{t}, t\right), \Psi\left(X_{t}, t\right)$, and $g\left(X_{t}, t\right)$ are convex functions.

To visualize the impact of BGC stochastic processes, 1000 Itô diffusions were simulated both with and without BGC, with unit diffusion coefficient $\sigma\left(X_{t}, t\right)$ for negative, zero, and positive drift $\mu\left(X_{t}, t\right)$ coefficients. Figure 1 shows this when $\mu\left(X_{t}, t\right)=0, \sigma\left(X_{t}, t\right)=1$, and $\Psi\left(X_{t}, t\right)$ is the parabolic cylinder, so that one can see the hidden upper barrier $\mathfrak{B}_{U}$ and hidden lower barrier $\mathfrak{B}_{L}$ emerge more clearly than is the case when using other coefficients. 


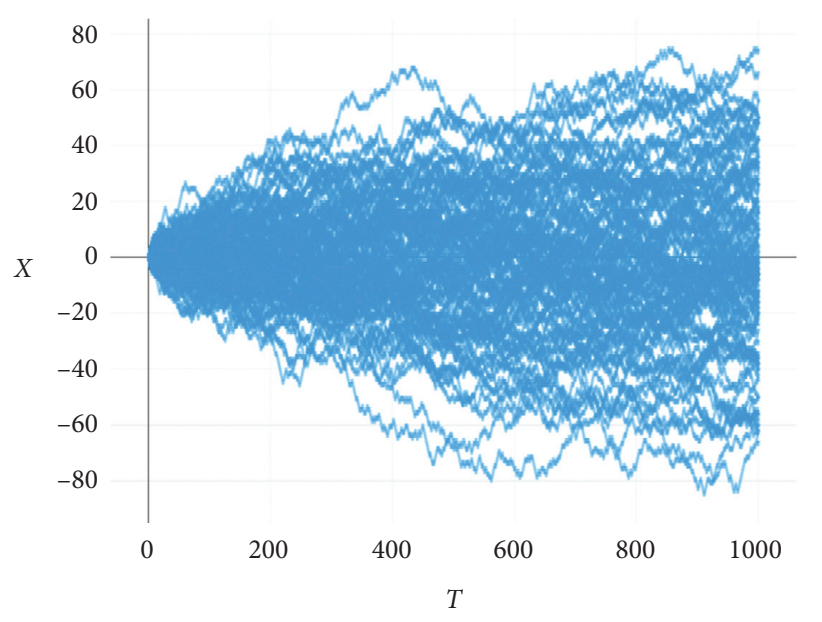

(a)

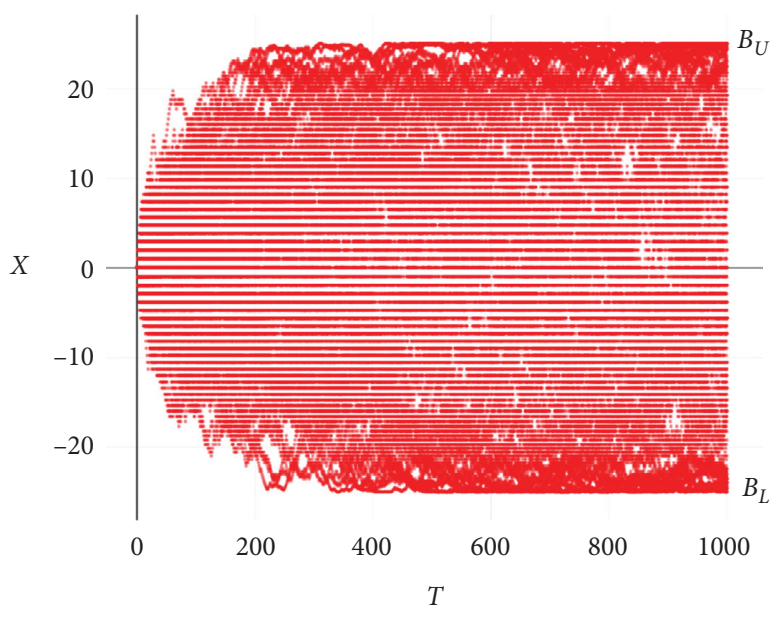

(b)

Figure 1: Itô diffusions with \& without BGC. The zero drift in (a) is constrained in (b) the more it deviates from the origin, causing the hidden reflective upper barrier $\mathfrak{B}_{U}$ and hidden reflective lower barrier $\mathfrak{B}_{\mathbf{L}}$ to emerge, together with horizontal bands to form due to the discretization effect of BGC.

Remark 1. From Figure 1, we immediately see the significance and applications of this research by being able to constrain stochastic processes within two reflective barriers. However, unlike the existing research in this established field, BGCSPs do not specify the barriers upfront, only the constraining mechanism of $\Psi\left(X_{t}, t\right)$. So, for a rough example, rather than central banks constraining their interest rates directly between two predefined (and sometimes illadvised) levels, BGCSPs just require how the constraining is to occur. The barriers emerge as the extremes of where the process can drift towards with increasing resistance.

Remark 2. The drift $f\left(X_{t}, t\right)$ and diffusion (or volatility) $g\left(X_{t}, t\right)$ terms reflect the instantaneous mean and standard deviation, respectively. It must also be noted from Figure 1 that even when a generalized Itô diffusion is reduced to a single Wiener process by setting $f\left(X_{t}, t\right)=0$ and $g\left(X_{t}, t\right)=1$, BGC still impacts the stochastic process.

It is from these observations that an alternative definition to (3) can be stated as follows.

Definition 2 (Definition II of BGC Stochastic Processes). For a complete filtered probability space $\left(\Omega, \mathscr{F},\{\mathscr{F}\}_{t \geq 0}, \mathbb{P}\right)$ and a BGC function $\Psi(x): \mathbb{R} \longrightarrow \mathbb{R}, \forall x \in \mathbb{R}$, the corresponding BGC Itô diffusion can be expressed as

$$
\mathrm{d} X_{t}=f\left(X_{t}, t\right) \mathrm{d} t+(g\left(X_{t}, t\right) \underbrace{-\operatorname{sgn}\left[X_{t}\right] \Psi\left(X_{t}, t\right)}_{\text {BGC }}) \mathrm{d} W_{t},
$$

where $\operatorname{sgn}[x]$ is defined in the usual sense, $f\left(X_{t}, t\right)$ is the drift term, $g\left(X_{t}, t\right)$ is the diffusion term, $\Psi\left(X_{t}, t\right)$ is the constraining term, and $f\left(X_{t}, t\right), g\left(X_{t}, t\right)$, and $\Psi\left(X_{t}, t\right)$ are convex functions.

Remark 3. In the work of Taranto et al. [1], only 2 decimal places were used, and some readers may argue that this is a low level of precision for simulation results to be robust. To show that the discretization effect present in BGC stochastic processes is not due to such rounding errors, all our simulations were rerun to ten times more precision (i.e., to 20 decimal places), and it was found that the discretization or banding effect of BGC was still present, so it is a real phenomenon. More shall be discussed about this in the Results and Discussion section.

This paper will answer two main objectives:

(1) What are the key properties of $\Psi\left(X_{t}, t\right)$ which are relevant in BGC stochastic processes?

(2) What is the formula for the hidden reflective lower barrier $\mathfrak{B}_{L}$ and the hidden reflective upper barrier $\mathfrak{B}_{U}$ in relation to $\Psi\left(X_{t}, t\right)$ ?

Before these objectives are addressed in the Methodology section, the relevant research is examined.

\section{Literature Review}

Constraining Discrete Random Walks. Constrained stochastic processes have been applied to game theory (Feller [2]) and conditional Markov chains of this type have also been applied to biology, branching processes (Ferrari et al. [3]), molecular physics (Novikov et al. [4]), medicine (Bell [5]), and queuing theory (Böhm and Gopal [6]) to name a few. Weesakul [7] discussed the classical problem of random walks restricted between a reflecting barrier and an absorbing barrier. Lehner [8] studied 1-dimensional random walks with a partially reflecting barrier using combinatorial methods. Gupta [9] introduced the concept of a multiple function barrier (MFB), where a state can either absorb, reflect, let through (transmit), or hold for a moment along with its corresponding probabilities. Dua et al. [10] found the bivariate generating functions of the probabilities of a random variable reaching a certain state under different 
conditions. Percus [11] considered asymmetric random walks, with one or two boundaries, on a 1-dimensional lattice. El-Shehawey [12] obtained absorption probabilities at the boundaries for random walks between one or two partially absorbing boundaries, using conditional probabilities.

Constraining Continuous Wiener Processes. Dirichlet studied the first boundary value problem, for the Laplace equation, proving the uniqueness of the solution and this type of problem in the theory of partial differential equations (PDEs). This was later named the Dirichlet problem after him (Gowers et al. [13]). Problems expressed within this framework were studied as early as 1840 by C.F. Gauss and then by Dirichlet [14]. Kurtz [15] formulated a means for constraining Markov processes. L'epingle [16] expanded upon previous research on barriers, which included boundary behavior of constrained Wiener processes between reflecting and repellent barriers. Majumdar et al. [17] derived the time taken to reach the maximum for a variety of constrained Wiener processes. Ormeci et al. [18] examined the constraining of Wiener processes via impulse control. Budhiraja and Dupuis [19] added necessary and sufficient conditions for the stability of such constrained processes. The same authors studied large deviations for various metrics of reflecting Wiener processes under constraining (Budhiraja and Dupuis [20]). Kharroubi et al. [21] constrained the jumps of Backward SDEs.

Whilst BGCSPs are relatively new, they do have applications in many areas, the most prominent being mathematical finance, investment algorithms, and quantitative trading (Taranto and Khan [22-25]). These have so far been defined in terms of 1-dimensional discrete random walks and continuous Itô diffusions but extend to the $n$-dimensional space $\mathbb{R}^{n}$. The constraining of such complex processes under multiple dimensions, variables, features, attributes, or columns in the context of machine learning (ML) essentially can be reduced to stochastic optimization. For example, under ML techniques such as Artificial Neural Networks (ANN), Deep Learning (DL), and Artificial Intelligence (AI), which have been extensively used in algorithmic trading, they essentially boiled down to finding the global maximum or global minimum of an $n$-dimensional surface. The geometry of such surfaces is thus not just an academic mathematical exercise but one that can shed light on when such algorithms can work and also when they can suddenly become ineffective.

We are now in a position to examine the geometry of the random variable $X$ as constrained by $\Psi\left(X_{t}, t\right)$ and its ramifications for BGCSP.

\section{Methodology}

3.1. Convexity of BGC. From (4), we know that $\Psi\left(X_{t}, t\right)$ needs to be a convex function and, specifically, centered about the origin. This is to ensure that the constraining applies increasing monotonic resistance to the Itô diffusion in both directions (i.e., bidirectionally). For example, $\Psi(x, t)=e^{t}$ would not be sufficient because whilst $e^{t}$ is convex (as shown by having a line bisecting any two points on its curve), $e^{t}$ does not increase as $t \downarrow-\infty$ as it does when $t \uparrow+\infty$. This gives rise to the need for the following classification of convexity.

Definition 3 (types of convexity). If $\Psi(x): \mathbb{R} \longrightarrow \mathbb{R}$ and $\Psi(x) \in C^{2}$, then we can characterize (Zalinescu [26], Bauschke and Combettes [27]) its convexity as follows:

(1) $\Psi(x)$ is convex if and only if $\Psi^{\prime \prime}(x) \geq 0, \forall x \in \mathbb{R}$

(2) $\Psi(x)$ is strictly convex if and only if $\Psi^{\prime \prime}(x)>0$, $\forall x \in \mathbb{R}$

(3) $\Psi(x)$ is strongly convex if and only if $\Psi^{\prime \prime}(x) \geq m>0$, $\forall x \in \mathbb{R}$

We will require a new type of (subset) convexity for BGCSP.

Definition 4 (bidirectional convexity). If $\Psi(x): \mathbb{R} \longrightarrow \mathbb{R}$ and $\Psi(x) \in C^{2}$, then we can characterize its convexity as follows:

$\Psi(x)$ is bidirectionally convex if and only if $\Psi^{\prime \prime}(x)>0$ and $\Psi(x)=\Psi(-x), \forall x \in \mathbb{R}$.

To establish some use cases to explore the convex geometry of potential BGC functions for $\Psi(x, t)$, we plot their surfaces in Figures 2 and 3 and deduce which specific type of convexity definition is required for BGCSP.

We examine five main types of bidirectionally convex functions in $\mathbb{R}^{3}$, as shown in Figures 2 and 3:

(1) Use Case I was not adopted because it constrains the Itô diffusion evenly and uniformly but not rapidly enough with the unconstrained Itô diffusions' iterated logarithm bounds growth rate. This is to the point that the before and after BGC plots (see Figure 4(a)) look very similar and do not constitute a practical and worthwhile BGC process.

(2) Use Case II is definitely the ideal function for BGC and so we will dedicate much of the Methodology section and the Results and Discussion section to the parabolic cylinder.

(3) Use Case III examines how the standard convex $e^{x}$ will not suffice because $\Psi(x, t)$ needs to be "Bidirectional"; that is, it must be a mirror reflection about the origin $X=0$ all the time. Hence, $e^{x}+e^{-x}$ was used, yet, due to the fast growing nature of the exponential function, it constrains the Itô process too much for it to be a useful function for BGC (even when it is scaled down by a constant $\omega$ or many other possible variations of $e^{ \pm x}$ ), as will be elaborated further in the Results and Discussion section (Figure 4(b)).

(4) Use Case IV was also presented here because it is a transition from no constraining to a gradual parabolic cylinder. Such a surface was proposed for applications in which Itô diffusions not constrained so much initially and which become increasingly more constrained over time are required. However, as will be detailed further in the Results and 


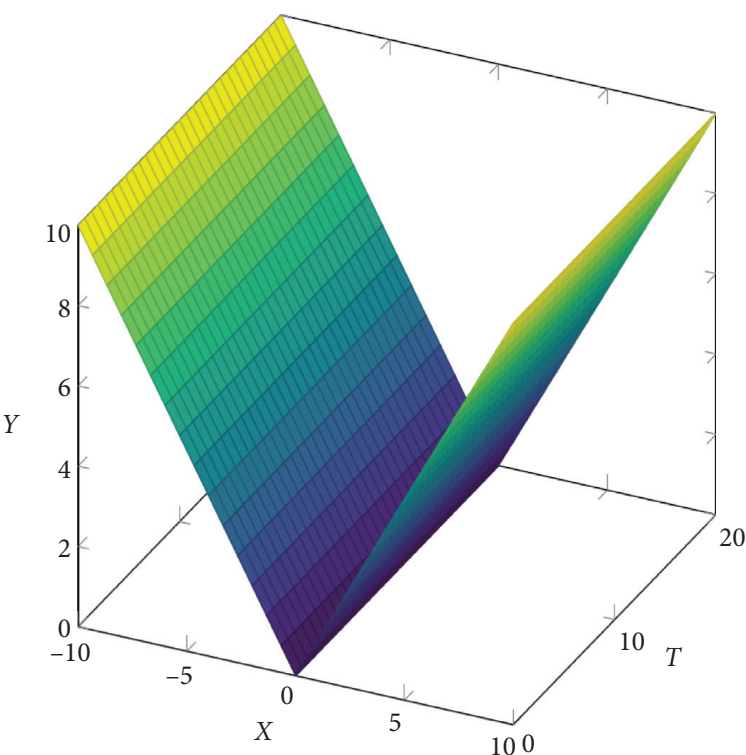

(a)

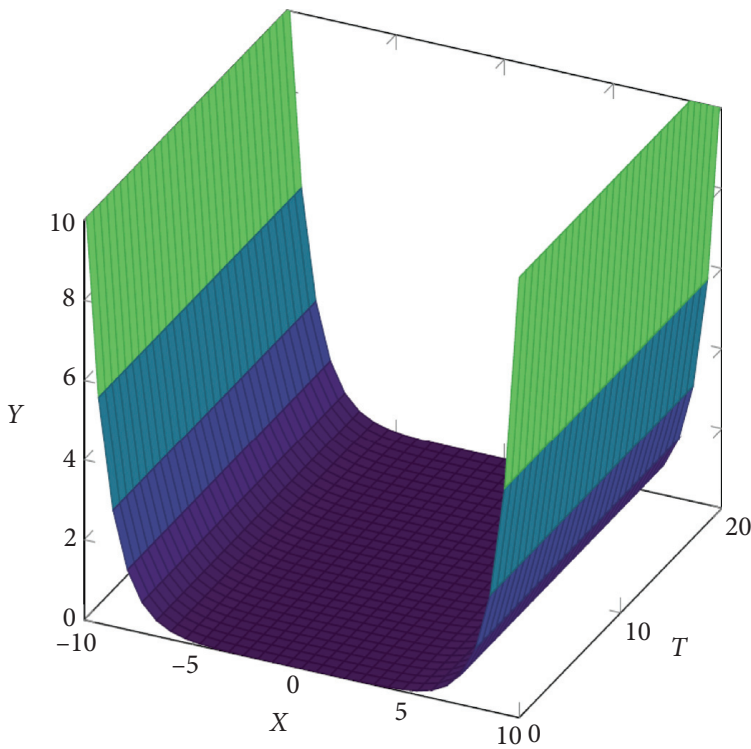

(c)

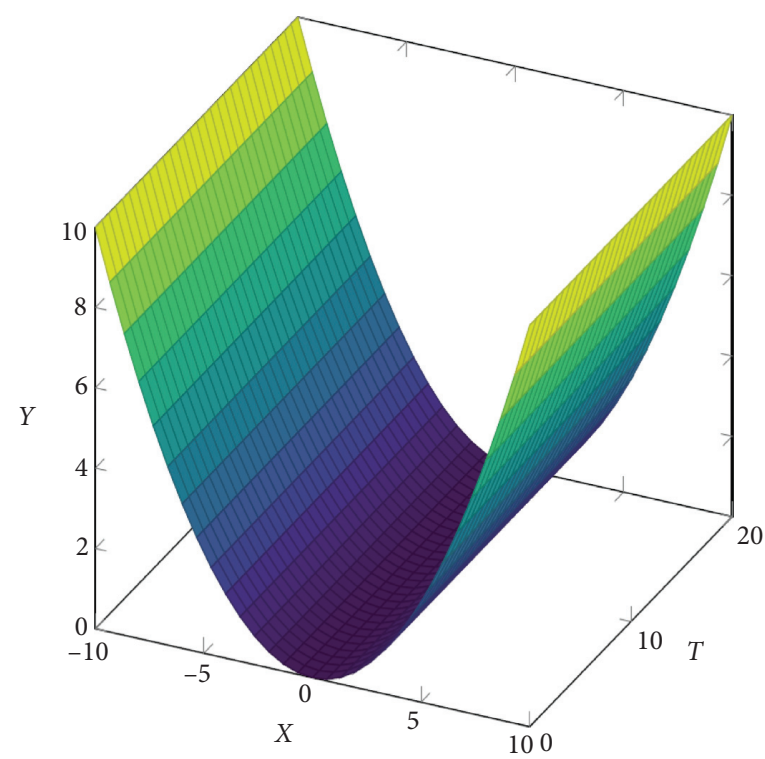

(b)

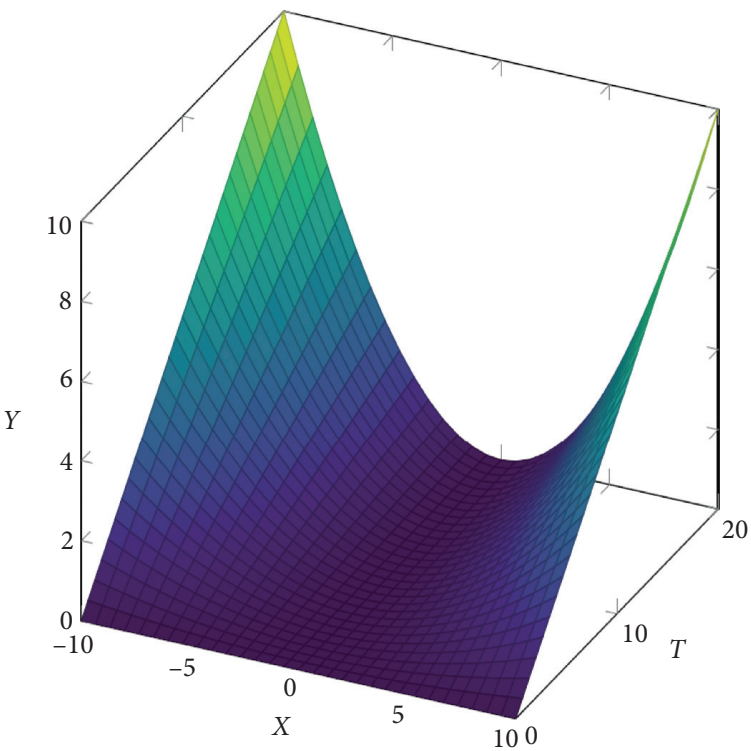

(d)

FIgURE 2: Use Cases I-IV: surface plots of potential convex BGC function $\Psi(x, t)$. (a) Use Case I: wedge. (b) Use Case II: parabolic cylinder. (c) Use Case III: double exponential cylinder. (d) Use Case IV: hybrid of flat plane and parabolic cylinder.

Discussion section (Figure 4(c)), this did not produce the hidden barriers that bound an Itô diffusion from both above and below.

(5) Use Case $\mathrm{V}$ is the final example that is worth discussing, as shown in Figure 3.

From Figure 3, we note that $y=x^{2 n}, \forall n \in \mathbb{N}$, will always result in a polynomial cylinder as $2 n$ will always be an even exponent. For odd exponents $y=x^{2 n+1}, \forall n \in \mathbb{N}$, one can simply replace this with $y=|x|^{2 n+1}$. In general, $y=|x|^{n}$ will be a polynomial cylinder that will always be convex and "Bidirectional," $\forall n \in \mathbb{N}$. As will be elaborated in the Results and Discussion section in Figure 4(d), the polynomial cylinder was not suitable as a BGC function for general unconstrained Itô diffusions but can be scaled to suit one's specific unconstrained Itô diffusion.

Remark 4. It is clear by now that not any convex function can be appropriate for BGC. An example of this would be $y=x^{2}+t^{2}$, where it is clearly and bidirectionally convex but not constant or "cylindrical" over time and does not resemble any natural regime to constrain the stochastic processes uniformly over time. It is thus clear now that BGC requires the bidirectionally convex definition and, in particular, bidirectionally convex cylinders.

Having explored the nature of $X$ as determined by $\Psi(x, t)$ which lies in $\mathbb{R}^{3}$, we notice that our Itô process in $X$ 


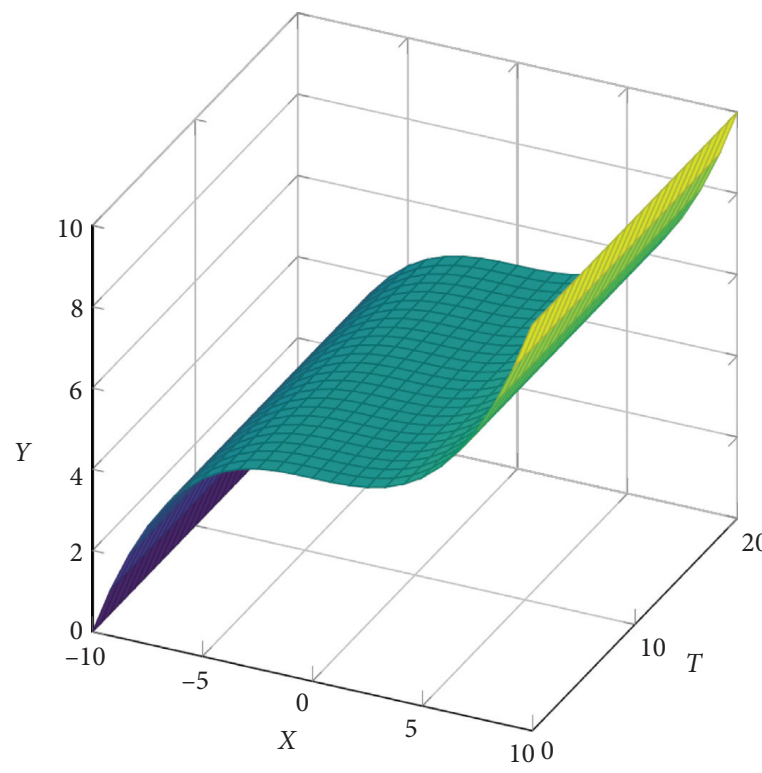

(a)

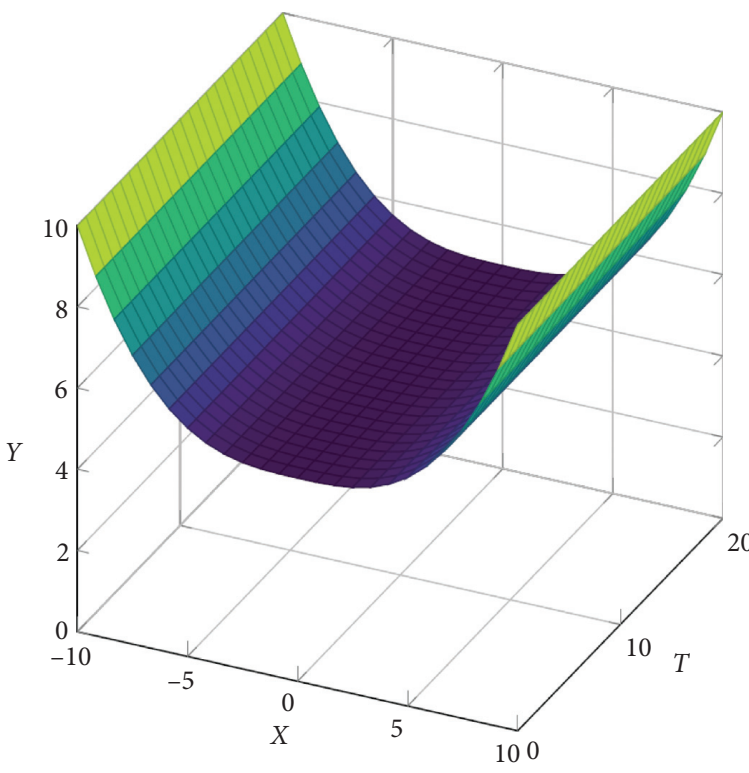

(b)

Figure 3: Technique to generate convex BGC polynomial cylinders (a). Cubic cylinder (b). Use Case V: spliced polynomial cylinder(s). (a) The cubic cylinder is concave for negative $\mathbf{X}$ values and convex for positive $\mathbf{X}$ values. (b) By forcing the negative values to be positive by the absolute value function, the entire function becomes convex and is Bidirectional of the polynomial cylinder variety (in this case a cubic cylinder). It is as if the positive part of the cubic cylinder was spliced in to replace the negative part. (a) $y=\left(x^{3} / w_{1}\right)+w_{2}, w_{1}=200, w_{2}=5$. (b) $y=\left(|x|^{3} / w_{1}\right)+w_{2}, w_{1}=200, w_{2}=5$.

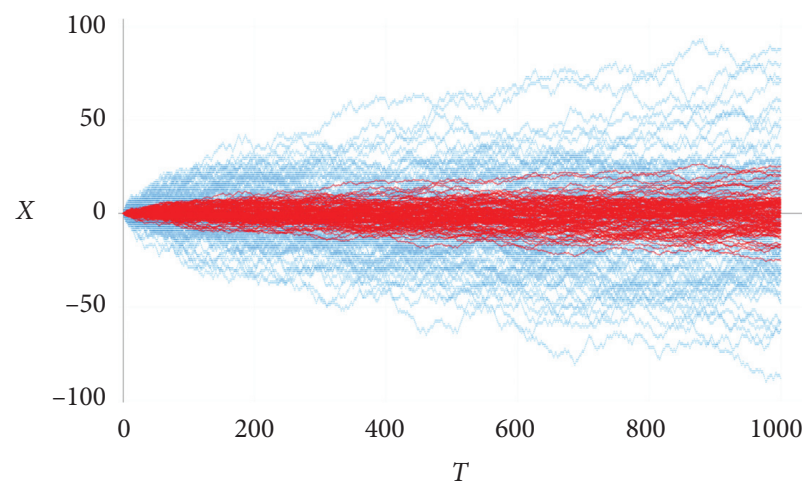

(a)

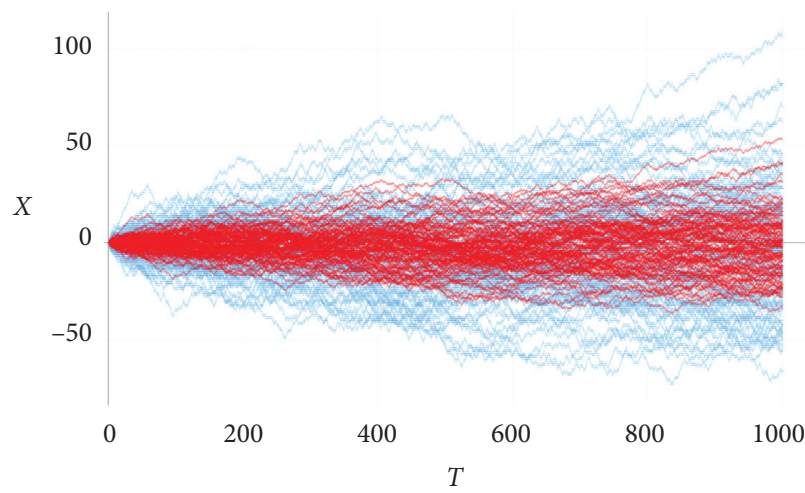

(c)

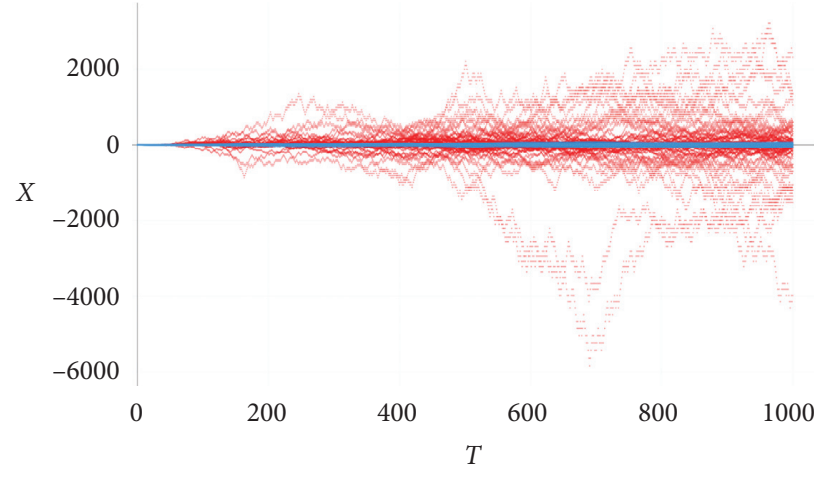

(b)

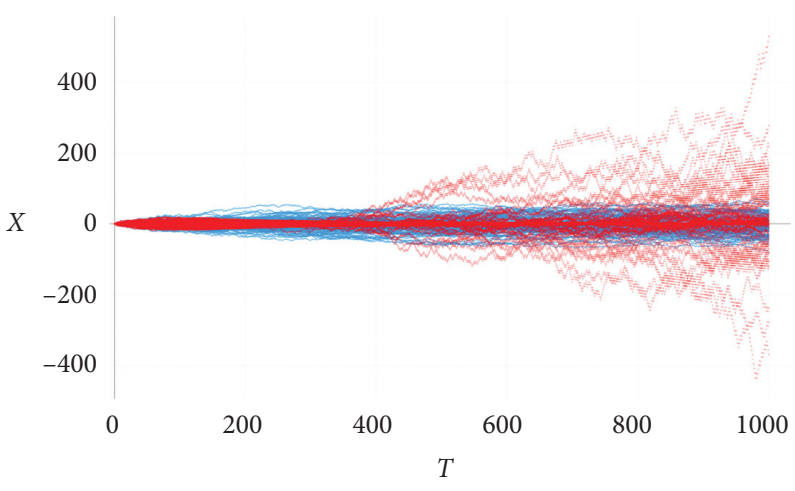

(d)

FIGURe 4: Potential convex candidates for $\Psi\left(X_{t}, t\right)$. Blue: without BGC; red: with BGC 10,000 unconstrained and 10,000 BGC Itô diffusions simulated. All the potential BGC functions $\Psi\left(\mathbf{X}_{\mathbf{t}}, \mathbf{t}\right)$ are not suitable for the standard Ito diffusions of BGCSP, even though they are bidirectionally convex, except for (c). (a) and (c) were rejected, since they do not constrain $\mathbf{X}_{\mathbf{t}}$ to constant hidden barriers. (b) and (d) were rejected because whilst there is some initial evidence of constant hidden barriers, they become unstable over time and explode to $\pm \infty$, faster than unconstrained Itô diffusions. (a) $y=|x|$. (b) $y=\left(e^{\infty}+e^{-\infty}\right) / \theta, \theta=2000$. (c) $y=\left(x^{2} t / \theta\right), \theta=200$. (d) $y=\left(|x|^{3} / \theta_{1}+\theta_{2}\right), \theta_{1}=200$. 
is a 1 -dimensional stochastic process in $\mathbb{R}$, where when it propagates over time, it does so in $\mathbb{R}^{2}$. The way we can see how the $3 \mathrm{D} \Psi(x, t)$ constrains the $1 \mathrm{D}$ Itô process in $2 \mathrm{D}$ is via the projection of $\Psi(x, t)$ onto the $\mathbb{R}^{2}$ plane via contour plots, as shown in Figures 5 and 6.

From Figure 5, it is much clearer to see how $\Psi(x, t)$ constrains the Itô process as $X_{t}$ propagates over time, where the lighter the colour, the greater the resistance and hence the greater the constraining impact due to BGC. This is also shown in the contour plots of Figure 6.

From these contour plots, we see how the convexity forms a series of decreasing semipermeable barriers (i.e., increasing reflection) on the Itô process. We now examine the effect that this has on the actual hidden reflective barriers, $\mathfrak{B}_{L}$ and $\mathfrak{B}_{U}$.

Remark 5. Note that an alternative to the $3 \mathrm{D}$ surface inducing the $2 \mathrm{D}$ contours is the $3 \mathrm{D}$ surface inducing the $3 \mathrm{D}$ and $2 \mathrm{D}$ vector fields, as shown in Figure 7.

From Figure 7 , we see that the constraining on $\mathbb{R}^{2}$ (specifically $\mathbb{R} \times \mathbb{R}_{+}$) can also be induced by the parabolic cylinder of $\Psi\left(X_{t}, t\right)$ and its associated vector field. As the Itô process propagates through the vector field, the greater the vector magnitudes, the greater the resistance force of reflection back to the origin.

This novel concept has been researched recently but in reverse by Simpson and Kuske [28], by modelling a constant variable into a random vector field to induce a stochastic process. Specifically, they show how a Flippov system near a switching manifold (due to the meeting of vector fields) attracts orbits or constant variables in the absence of randomness to create stochastic flow within the field.

3.2. Hidden Barriers of BGC Stochastic Processes. Whilst Figure 1(b) shows that the detailed nature of the hidden reflective BGC varies, we only plot $\mathfrak{B}_{L}$ and $\mathfrak{B}_{U}$ to better help derive the formulation of the barriers, as shown in Figure 8.

From Figure 8 , we see that the BGC hidden reflective barriers are regulated to one's desired distance from the origin by altering the $A$ parameter and are regulated in their climb rate from the origin to the barrier by altering the $\theta$ parameter.

Remark 6. Note that these barriers are not the traditional constant reflective barriers such as $X=a$ or $X=b$ because the BGC Itô diffusions are bounded within these nonconstant barriers even as they depart from the origin (i.e., the unconstrained Itô diffusions exceed these barriers, even near the origin), hence the initial curvature in the barriers. This is generalized in the following theorem.

Theorem 1 (Hidden Barriers of BGC Stochastic Processes). For a complete filtered probability space $\left(\Omega, \mathscr{F},\{\mathscr{F}\}_{t \geq 0}, \mathbb{P}\right)$ and a BGC function $\Psi(x): \mathbb{R} \longrightarrow \mathbb{R}$, $\forall x \in \mathbb{R}$, the corresponding BGC Itô diffusion is expressed as

$$
\mathrm{d} X_{t}=(\overbrace{f\left(X_{t}, t\right) \underbrace{-\operatorname{sgn}\left[X_{t}\right] \Psi\left(X_{t}, t\right)}_{\text {BGC }}}^{\mu\left(X_{t}, t\right)}) \mathrm{d} t+\overbrace{g\left(X_{t}, t\right)}^{\sigma\left(X_{t}, t\right)} \mathrm{d} W_{t},
$$

for $t \in[0, T]$, where $\operatorname{sgn}[x]$ is the sign function defined in the usual sense, $f\left(X_{t}, t\right)$ is a drift term, $\Psi\left(X_{t}, t\right)$ is the BGC term, $g\left(X_{t}, t\right)$ is the diffusion term, and $f\left(X_{t}, t\right), \Psi\left(X_{t}, t\right)$, and $g\left(X_{t}, t\right)$ are bidirectionally convex functions. Then the hidden lower barrier $\mathfrak{B}_{L}$ and hidden upper barrier $\mathfrak{B}_{U}$ are defined $\forall \omega \in \Omega$ as

$$
\begin{aligned}
\mathfrak{B}_{L} & =\int_{0}^{\infty} \inf _{0 \leq t \leq T}\left\{W_{t}(\omega)\right\} \mathrm{d} t, \\
\mathfrak{B}_{U} & =\int_{0}^{\infty} \sup _{0 \leq t \leq T}\left\{W_{t}(\omega)\right\} \mathrm{d} t,
\end{aligned}
$$

and they are estimated by

$$
\begin{aligned}
& \mathfrak{B}_{L} \geq-A\left(1-e^{-\theta T}\right), \\
& \mathfrak{B}_{U} \leq A\left(1-e^{-\theta T}\right)
\end{aligned}
$$

where $A, \theta \in \mathbb{R}$ are constants, $A$ is the distance from the origin to the barrier(s), and $\theta$ is the rate of growth towards the barrier(s).

Proof. To some readers, (7) is obvious just by looking at Figure 8 (a) simply because the function must asymptote horizontally (exponentially) towards the barrier(s). However, this does not constitute a proof because we have a stochastic (and not a deterministic) process. Assume for a moment that the above BGC SDE is a simpler object in which $\mu\left(X_{t}, t\right)$ and $\sigma\left(X_{t}, t\right)$ are constant, where the drift function $\mu(x): \mathbb{R} \longrightarrow \mathbb{R}$ and the diffusion function $\sigma(x): \mathbb{R} \longrightarrow \mathbb{R}, \forall x \in \mathbb{R}$, in the limit approach the typical constant expressions for the drift and diffusion coefficients, $\lim _{x \longrightarrow \infty} \mu(x) \longrightarrow \mu$, and $\lim _{x \longrightarrow \infty} \sigma(x) \longrightarrow \sigma$. We now start to see the resemblance between our BGC Itô process and the simpler Ornstein-Uhlenbeck process (OUP):

$$
\mathrm{d} X_{t}=\overbrace{\kappa\left(\alpha-X_{t}\right)}^{\mu\left(X_{,}, t\right)} \mathrm{d} t+\sigma \mathrm{d} W_{t},
$$

where $\alpha$ is the long-term mean and $\kappa$ is the "attraction rate" or speed of mean-reversion, both of which are constants, as depicted in Figure 9.

From Figure 9, we can see that the total number of paths (as shown in blue and red) is preserved in each of the three schemes. (4) is (3) repeated for convenience and (4) is similar to (7), where $\kappa\left(\alpha-X_{t}\right)$ in OUP is replaced by $\kappa(\alpha-$ $\left.\operatorname{sgn}\left[X_{t}\right] \Psi\left(X_{t}, t\right)\right)$ in BGCSP (with $\kappa=1$ and $\alpha=f\left(X_{t}, t\right)$ ), which caters for a much wider set of possible paths than is possible with OPU and yet BGCSP has $\operatorname{sgn}\left[X_{t}\right] \Psi\left(X_{t}, t\right)$ instead of $X_{t}$. Multiplying (7) by $e^{k t}$ and expanding gives 


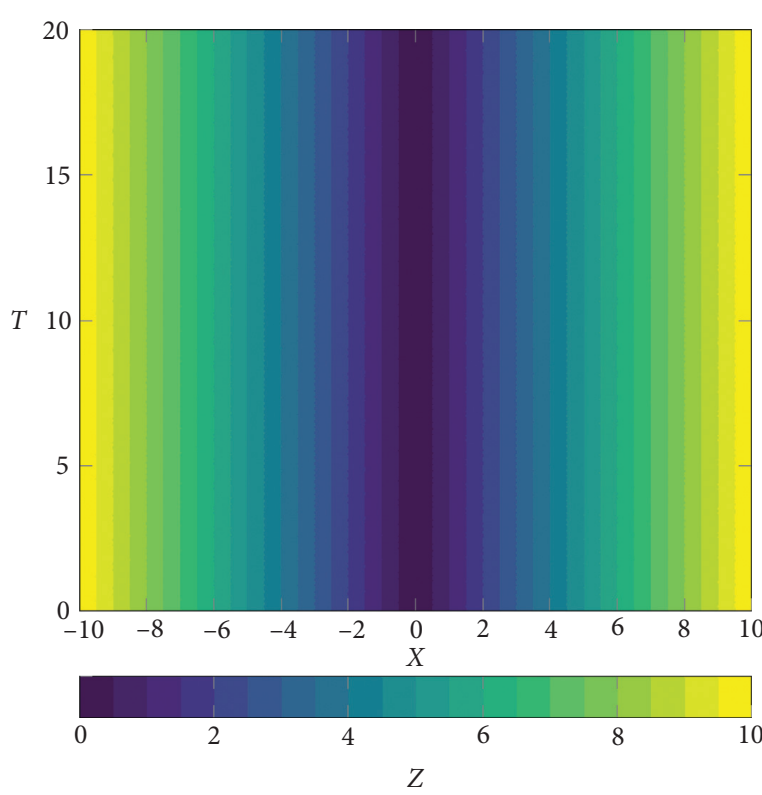

(a)

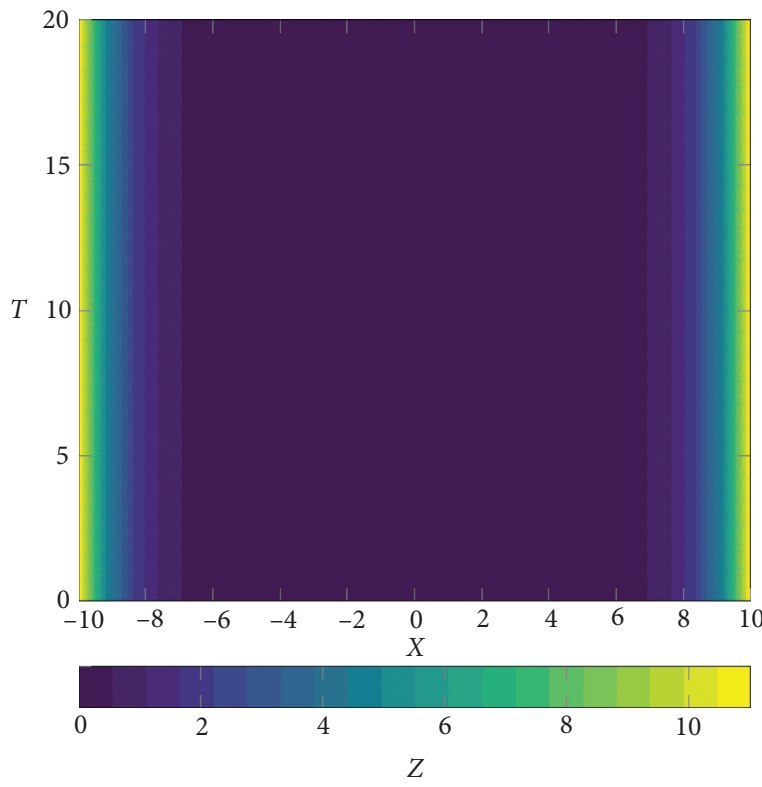

(c)

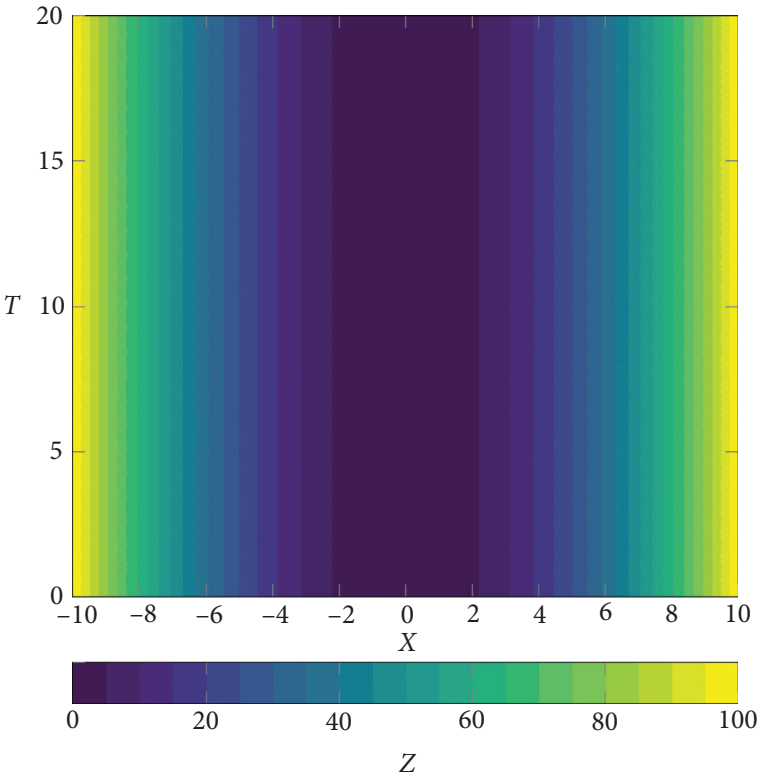

(b)

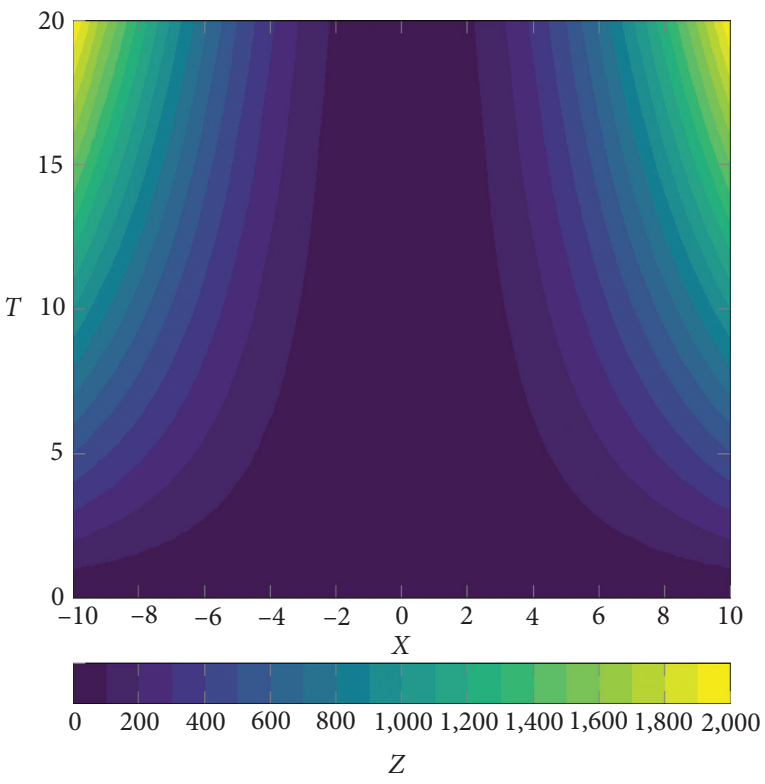

(d)

FIGURE 5: Contour plots of main use case candidates for BGC. (a) $y=|x|$. (b) $y=\left(x^{2} / w\right), w=10$. (c) $y=\left(\left(e^{\infty}+e^{-\infty}\right) / w\right), w=2000$. (d) $y=\left(x^{2} t / w\right), w=200$. The $\mathbf{Z}$ scale shows the 3D surface's height as it is mapped onto $\mathbb{R}^{2}$ and $\omega$ is a scaling function. (a) Use Case I: contour of a wedge. (b) Use Case II: contour of a parabolic cylinder. (c) Use Case III: contour of a double exponential cylinder. (d) Use Case IV: contour of a hybrid of flat plane and parabolic cylinder. 


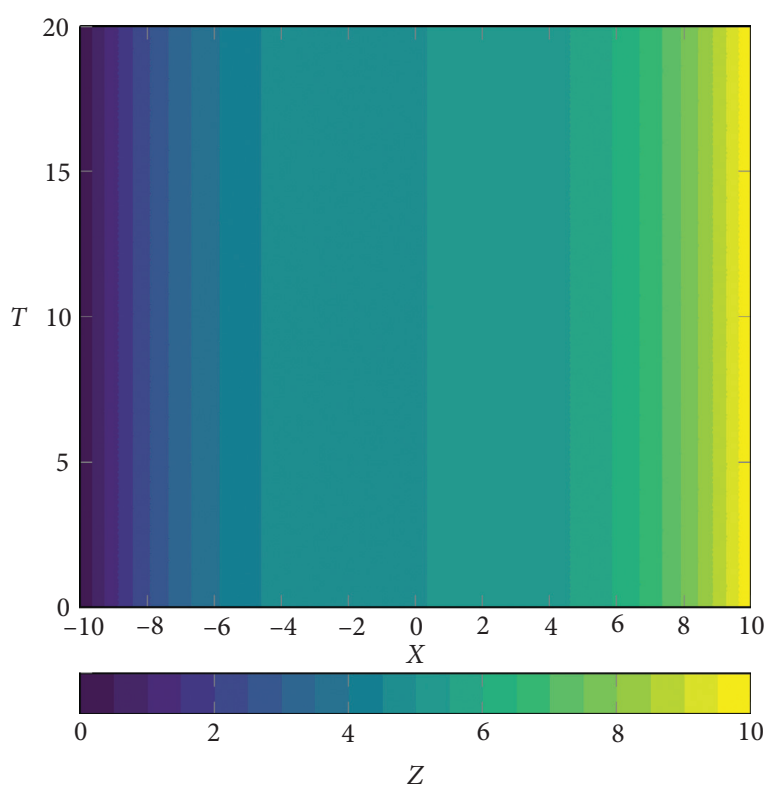

(a)

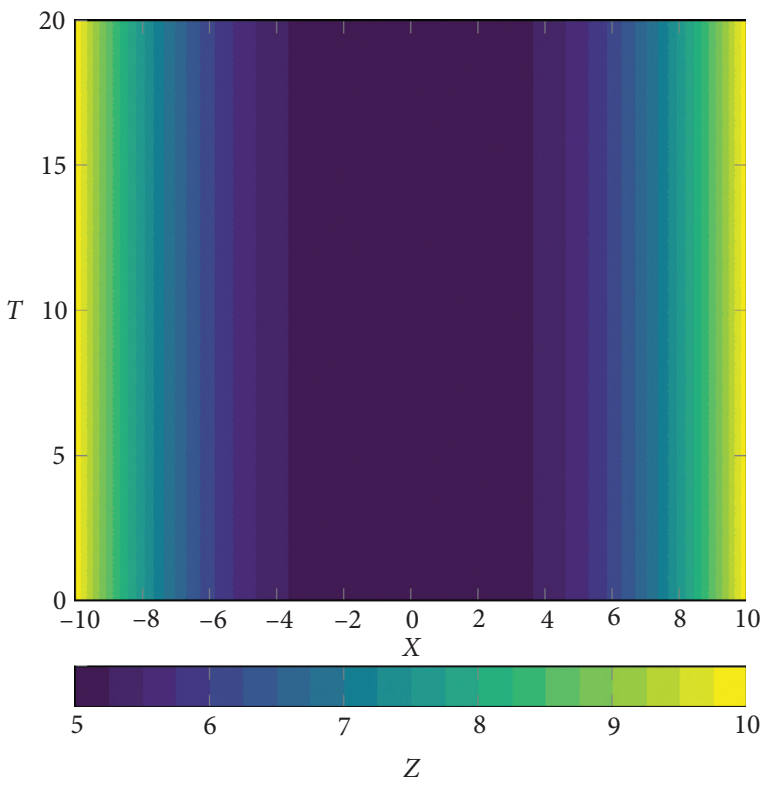

(b)

Figure 6: Contour plots as $y=x^{3}$ is made Bidirectional. The $\mathbf{Z}$ scale shows the $3 \mathrm{D}$ surface's height as it is mapped onto $\mathbb{R}^{2}$. (a) $\mathbf{y}=\mathbf{x}^{3}:$ we can see how negative values of $\mathbf{X}$ lead to a concave contour, whereas positive values of $\mathbf{X}$ lead to a convex contour, due to the odd power of $\mathbf{x}^{3}$. (b) Use Case V: by taking the absolute value of $\mathbf{x}$ before passing it through $\mathbf{x}^{3}$, we see that the contour plot is convex in both directions of $\mathbf{X}$, that is, is Bidirectional. (a) $y=\left(x^{3} / w_{1}\right)+w_{2}, w_{1}=200, w_{2}=5$. (b) $y=\left(|x|^{3} / w_{1}\right)+w_{2}, w_{1}=200, w_{2}=5$.

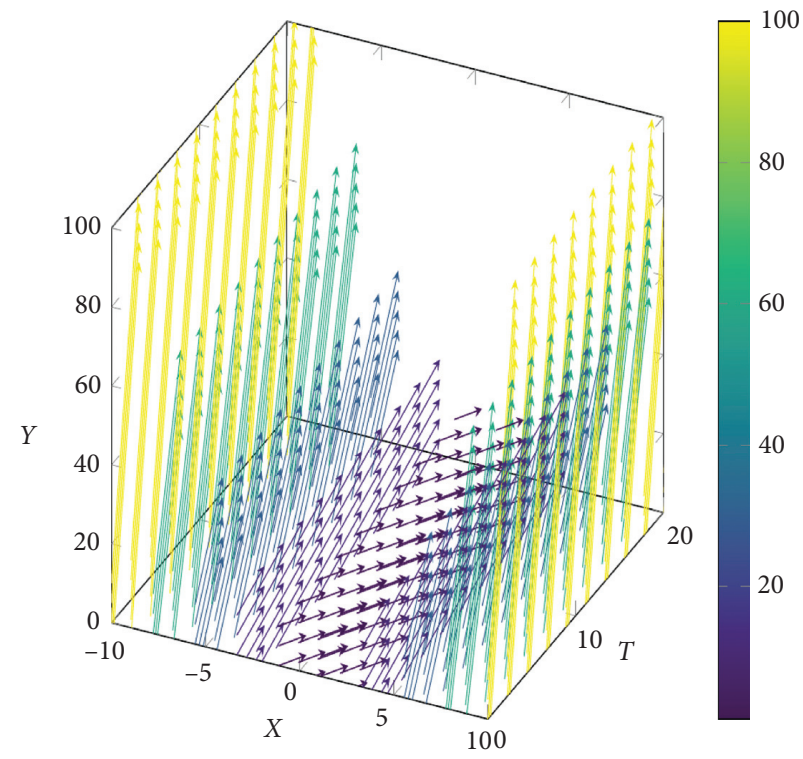

(a)

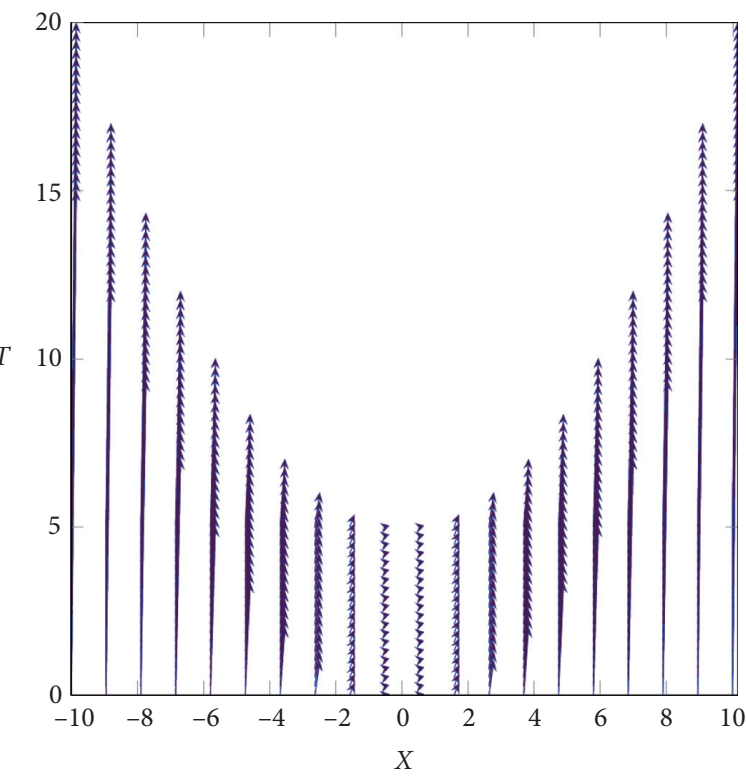

(b)

Figure 7: Vector fields induced by BGC constraints. Just as in Figure 5(b) for the 2D contour map induced by the 3D parabolic cylinder surface, here the $3 \mathrm{D}$ vector field in (a) also induces a constraining force on the $2 \mathrm{D}$ vector plot in (b). (a) BGC vector field in $\mathbb{R}^{3}$. (b) BGC vector field in $\mathbb{R}^{2}$. 


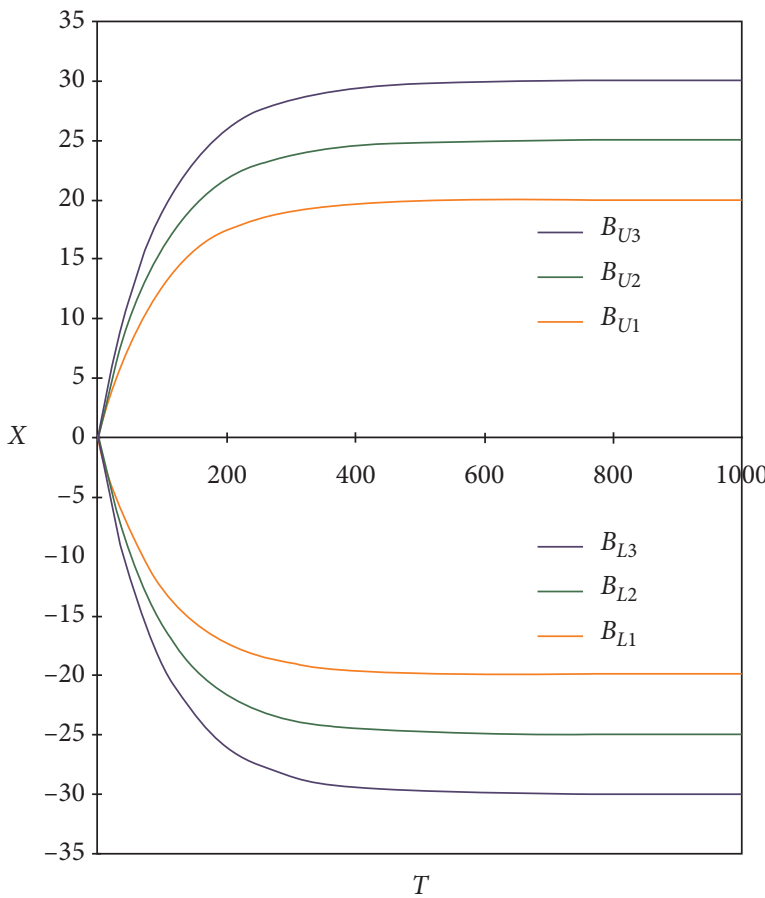

(a)

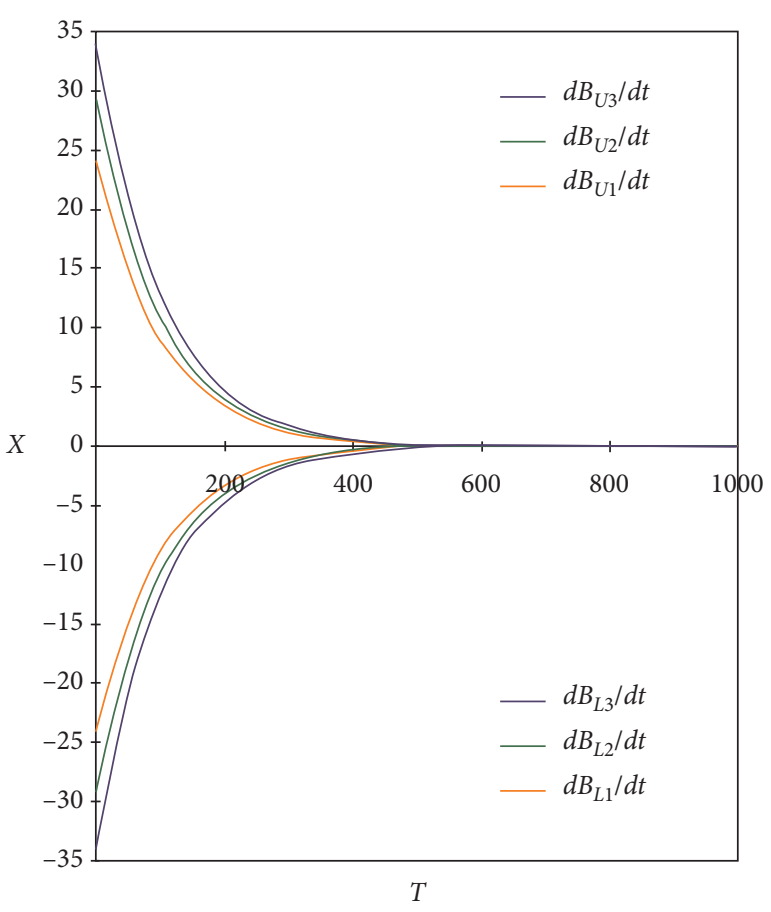

(b)

Figure 8: BGC hidden reflective barriers identified. Orange: $\mathbf{A}= \pm 25$, green: $\mathbf{A}= \pm 30$, purple: $\mathbf{A}= \pm 35$. (a) $\mathfrak{B}_{\mathbf{L}}=-\mathbf{A}\left(1-\mathbf{e}^{-\theta \mathbf{T}}\right)$, $\mathfrak{B}_{\mathbf{U}}=\mathbf{A}\left(1-\mathbf{e}^{-\theta \mathbf{T}}\right), \theta=0.01$. (b) The rate of change of the barriers, that is, $\left(\mathbf{d} \mathfrak{B}_{\mathbf{L}} / \mathbf{d t}\right)$ and $\left(\mathbf{d} \mathfrak{B}_{\mathbf{U}} / \mathbf{d t}\right)$.

$$
\begin{aligned}
e^{\kappa t} \mathrm{~d} X_{t}+\kappa e^{\kappa t} X_{t} \mathrm{~d} t & =\kappa \alpha e^{\kappa t} \mathrm{~d} t+\sigma e^{\kappa t} \mathrm{~d} W_{t} \\
d\left(e^{\kappa t} X_{t}\right) & =\kappa \alpha e^{\kappa t} \mathrm{~d} t+\sigma e^{\kappa t} \mathrm{~d} W_{t} \\
\int_{0}^{T} \mathrm{~d}\left(e^{\kappa t} X_{t}\right) & =\int_{0}^{T} \kappa \alpha e^{\kappa t} \mathrm{~d} t+\int_{0}^{T} \sigma e^{\kappa t} \mathrm{~d} W_{t} \\
e^{\kappa T} X_{T}-e^{0} X_{0} & =\kappa \alpha \frac{e^{\kappa T}-e^{0}}{\kappa}+\sigma \int_{0}^{T} e^{\kappa t} \mathrm{~d} W_{t} \\
X_{T} & =X_{0} e^{-\kappa T}+\alpha\left(1-e^{-\kappa T}\right)+\sigma \int_{0}^{T} e^{-\kappa(T-t)} \mathrm{d} W_{t} .
\end{aligned}
$$

Having solved the OUP SDE, we wish to determine where $X_{t}$ is most likely to be for $t=T$. Taking the expectation of both sides,

(9)

$$
\mathbb{E}\left[X_{T}\right]=\mathbb{E}\left[X_{0} e^{-\kappa T}+\alpha\left(1-e^{-\kappa T}\right)+\sigma \int_{0}^{T} e^{-\kappa(T-t)} \mathrm{d} W_{t}\right]=X_{0} e^{-\kappa T}+\alpha\left(1-e^{-\kappa T}\right)
$$

as $e^{-\kappa(T-t)}$ is deterministic. Since $X_{0}=0$ for all BGCSPs, we will set all OUPs to start from the origin, giving

$$
\mathbb{E}\left[X_{T}\right]=\alpha\left(1-e^{-\kappa T}\right) .
$$

We now have a stochastic argument that is a basis to justify (6). Assume that, for greatest generality,

$$
\begin{aligned}
& \mathfrak{B}_{L}=-A\left(1-e^{-\theta T}\right)+C \\
& \mathfrak{B}_{U}=A\left(1-e^{-\theta T}\right)+C
\end{aligned}
$$

We know that since $X_{t}$ is Bidirectional, it is symmetric about the origin and so $C=0$. By comparing (12) with (11), we can see that

$$
A\left(1-e^{-\theta T}\right) \sim \alpha\left(1-e^{-\kappa T}\right), \quad A \sim \alpha, \theta \sim \kappa,
$$




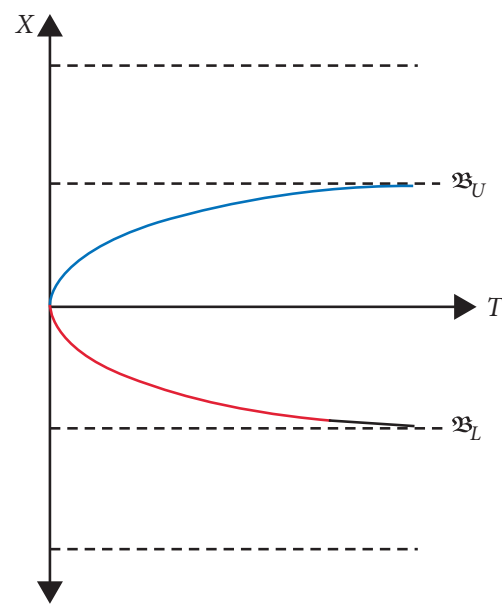

(a)

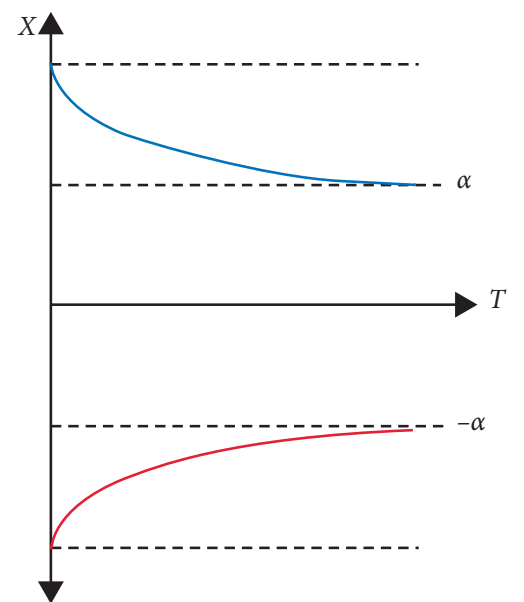

(b)

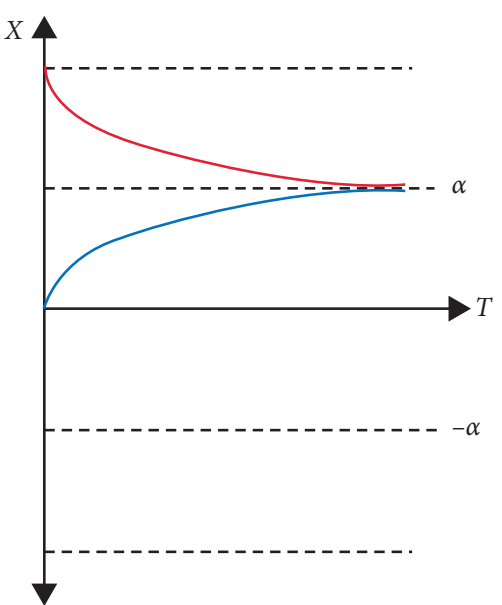

(c)

FIGURE 9: BGC hidden reflective barriers relation to Ornstein-Uhlenbeck process. (a) Idealized BGC process: BGCSP involves Itô processes that start at an initial position $\mathbf{x}_{0}=0$, where the further they drift away from the long run mean $\mathbf{X}=0$, the slower they will approach the hidden barriers $\mathfrak{B}_{\mathbf{L}}$ and $\mathfrak{B}_{\mathrm{U}}$, where $\mathbf{x}_{0}>\mathfrak{B}_{\mathrm{L}}$ and $\mathbf{x}_{0}<\mathfrak{B}_{\mathrm{U}}$. (b) Idealized hybrid between BGCSP and OUP: via the reflection principle, we can better see the transition between the other two paradigms. (c) Idealized Ornstein-Uhlenbeck process: OUP involves Itô processes that can start at any initial position $\mathbf{x}_{0} \in \mathbb{R}$ and will "gravitate" to the long-term mean $\alpha$.

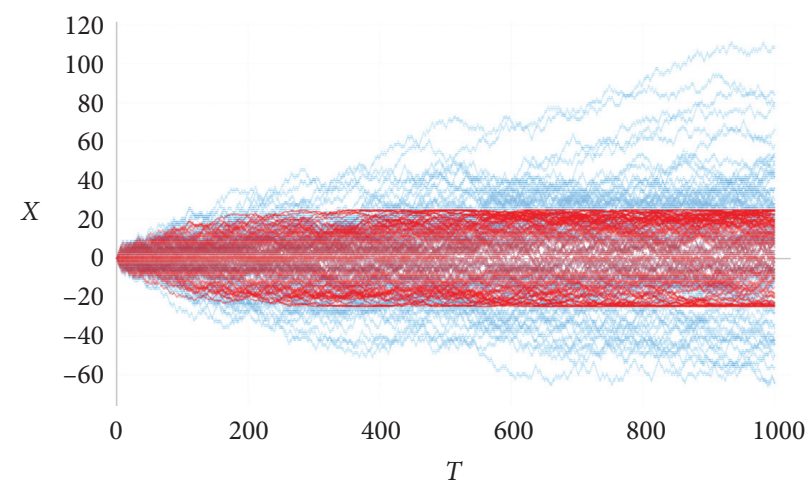

FIGURE 10: Ideal selection of $\Psi(x, t)$ for BGC. Blue: unconstrained Itô process; red: BGC Itô process. 10,000 unconstrained and 10,000 BGC Itô diffusions simulated for $\omega=100$ in $\Psi\left(\mathbf{X}_{\mathbf{t}}, \mathbf{t}\right)=\left(\mathbf{x}^{2} / \omega\right)$. As more and more BGC Itô processes are added to the plot, the ideal hidden reflective barriers of BGCSP emerge, confirming that $\mathfrak{B}_{\mathbf{L}}=-\mathbf{A}\left(1-\mathbf{e}^{-\theta \mathbf{T}}\right)$ and $\mathfrak{B}_{\mathbf{U}}=\mathbf{A}\left(1-\mathbf{e}^{-\theta \mathbf{T}}\right)$, where $\mathbf{A}$ controls the position of the barrier(s) and $\theta$ controls the speed at which the BGC Itô process reaches the hidden reflective barrier. In this plot, $\mathbf{A}=25$ and $\theta=0.01$, but these can be altered to suit one's BGC needs.

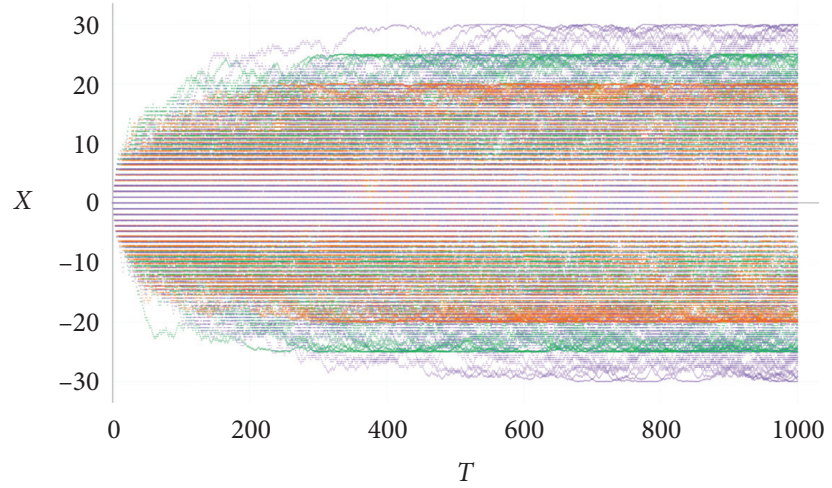

Figure 11: Parabolic cylinder expression of $\Psi\left(X_{t}, t\right)$ for three different values of $A$ for $\mathfrak{B}_{L}$ and $\mathfrak{B}_{U}$. Orange: inner-most barriers; green: middle barriers; purple: outer-most barriers. 10,000 BGC Itô diffusions simulated for various values of $\omega$ in $\Psi\left(\mathbf{X}_{\mathbf{t}}, \mathbf{t}\right)=\left(\mathbf{x}^{2} / \omega\right)$. The general formula for a hidden reflective BGC barrier for a parabolic cylinder is further validated as $\mathfrak{B}_{\mathbf{L}}=-\mathbf{A}\left(1-\mathbf{e}^{-\theta \mathbf{T}}\right)$ and $\mathfrak{B}_{\mathbf{U}}=\mathbf{A}\left(1-\mathbf{e}^{-\theta \mathbf{T}}\right)$, where $\theta=0.01$ and $\mathbf{A}=25$ for orange, $\mathbf{A}=30$ for green, and $\mathbf{A}=35$ for purple. Note that, for $\Psi(\mathbf{x}, \mathbf{t})=\left(\mathbf{x}^{2} / \omega\right), \omega=80$ for orange, $\omega=100$ for green, and $\omega=120$ for purple. 


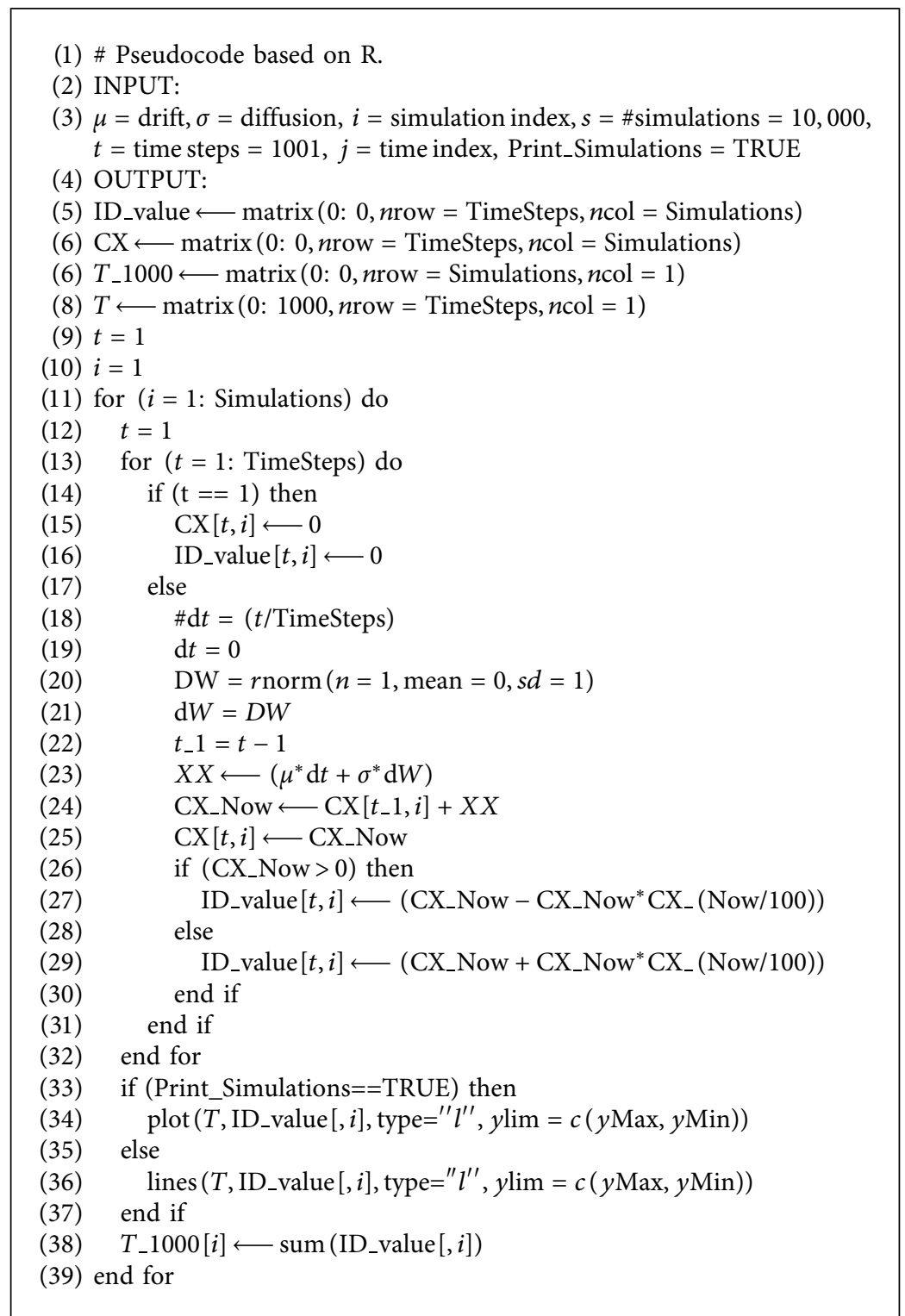

Algorithm 1: Bidirectional Grid Constrained (BGC) stochastic processes.

where $\sim$ signifies a weak association. Since BGCSPs do not "force" the Itô diffusion to the long-term mean $\alpha$, the time taken to reach $\alpha$ or $-\alpha$ under BGC would be greater than for OUP. Hence, in terms of distance,

$$
\begin{aligned}
& \mathfrak{B}_{L} \geq-A\left(1-e^{-\theta T}\right), \\
& \mathfrak{B}_{U} \leq A\left(1-e^{-\theta T}\right) .
\end{aligned}
$$

Remark 7. $A \sim \mu$ is intuitive because the greater the drift $|\mu|$, the greater $A$ and hence $|\mathfrak{B}|$, that is, $\mathfrak{B}_{L}$ and $\mathfrak{B}_{U}$. We also notice that the diffusion term $\sigma$ does not contribute as much to $|\mathfrak{B}|$. We also know by some experimentation that $\theta \in[0,1]$.

To extend [1] further, the BGCSP algorithm (Section 3.2) is derived and simulated in the Results and Discussion section, in Figures 4, 10, and 11 (Algorithm 1).

\section{Results and Discussion}

At this stage, we know that Use Cases I, III, IV, and V are not valid candidates for the correct type of convexity for general $\Psi\left(X_{t}, t\right)$, but, for various exotic forms of Itô diffusions, such as the Cox-Ingersoll-Ross (CIR) process, these use cases that may be sufficient to BGC the Itô process within two hidden barriers. We also have a theoretical appreciation of what other forms of $\Psi\left(X_{t}, t\right)$ are valid and invalid candidates.

On the other hand, we also know that Use Case II, the parabolic cylinder, is the ideal type of convexity for general Itô diffusions. To confirm this and further eliminate any remaining use cases, we simulate them in Figure 4.

From Figure 4, in (b) and (d), we see that a certain amount of constraining is occurring and that the BGC never exceeds the original simulation paths. However, we also see in (d) that whilst there is some BGC initially, after some time the hidden barriers are unstable. 
In (a) and (c), there is no real effective constraining, since the BGCSP is now hyperextended from the original simulation paths and so there are no hidden reflective barriers either. When $\Psi\left(X_{t}, t\right) \gg X_{t}$ (where $X_{t}$ is the unconstrained Ito diffusion and $\gg$ signifies domination), there will be a point in time where $X_{t}$ will flip over the origin and the contribution from the drift will be eclipsed by the $\Psi\left(X_{t}, t\right)$ term. We thus have $\Psi\left(X_{t}, t\right)$ becoming the dominant drift term that will explode the Itô diffusion beyond where the unconstrained Itô diffusion would reach, away from the origin.

We now examine the parabolic cylinder in far greater detail, as shown in Figure 10.

From Figure 10, we see that the hidden reflective BGC barriers can constrain the Itô diffusion(s) indefinitely as it is "trapped" within the barriers. This assumes that there are no sudden jumps (as is the case in jump-diffusion models) or changes in $X_{t}$ or in $\Psi\left(X_{t}, t\right)$. To examine this in even further detail, we simulate again for different parameters, as shown in Figure 11.

From Figure 11, there is a region about the time axis that not many simulation paths visit, supporting the notion that as the paths approach the hidden barriers, they end up being "trapped" near that boundary. Also notice how there is banding or discretization about various local times which get compressed the further they are from the origin. The local times seem to coincide or line up most near the time axis regardless of $\omega$.

\section{Conclusions}

This paper has extended the available research on BGCSPs by investigating the hidden geometry of the constraining BGC function(s). The parabolic cylinder was found to be the ideal constraining mechanism for the parameter $\Psi\left(X_{t}, t\right)$, for the most general unconstrained Itô diffusions. The constraining geometry must be convex but not any ordinary convex function will suffice. The novel "bidirectionally convex" definition was defined and adopted. The formulas for the lower hidden reflective barrier $\mathfrak{B}_{L}$ and the upper hidden reflective barrier $\mathfrak{B}_{U}$ were derived. This helps establish a linkage between $\Psi\left(X_{t}, t\right)$ of the form $\left(x^{2} / \omega\right)$ and the resulting $\mathfrak{B}_{L}$ and $\mathfrak{B}_{U}$. By solving the Ornstein-Uhlenbeck process (OUP), we have been able to show the linkage between OUP and the Langevin SDE, in relation to BGCSP's hidden barriers.

This research has applications in many fields, such as in finance where exchange rates can be constrained by "parabolic cylinder" monetary policies, such as "keep the AUD/ NZD exchange rate within a range by regulating the amount of Government debt, the more it approaches the range boundaries." Rather than prespecifying the boundaries, one can now specify just the transitions towards the unknown boundaries that can be estimated in advance. How the transition occurs is thus more important than what the final extremes (barriers) are. Future research in BGC can involve BGC of other important Itô diffusions from other research fields and finding estimates for the distribution of the first passage time (FPT) for when $\mathfrak{B}_{L}$ and $\mathfrak{B}_{U}$ are most likely to be first hit.

\section{Data Availability}

The simulated data used to support the findings of this study are available from the corresponding author upon request.

\section{Conflicts of Interest}

The authors declare that they have no conflicts of interest.

\section{Acknowledgments}

The first author was supported by an Australian Government Research Training Program (RTP) Scholarship. The authors would like to thank A/Professor Ron Addie of University of Southern Queensland for advice on refining this paper.

\section{References}

[1] A. Taranto, S. Khan, and R. Addie, "Iterated logarithm bounds of bi-diretional grid constrained stochastic processes," 2020, https://arxiv.org/abs/2103.02731.

[2] W. Feller, An Introduction to Probability Theory and Its Applications, Wiley International Publishing, Hoboken, NJ, USA, 3rd edition, 1968.

[3] P. A. Ferrari, S. Martínez, and P. Picco, "Existence of nontrivial quasi-stationary distributions in the birth-death chain," Advances in Applied Probability, vol. 24, no. 4, pp. 795-813, 1992.

[4] D. S. Novikov, E. Fieremans, J. H. Jensen, and J. A. Helpern, "Random walks with barriers," Nature Physics, vol. 7, no. 6, pp. 508-514, 2011

[5] G. Bell, "Models of carcinogenesis as an escape from mitotic inhibitors," Science, vol. 192, no. 4239, pp. 569-572, 1976.

[6] W. Böhm and M. Gopal, On Random Walks with Barriers and Their Application to Queues, p. 21, Forschungsberichte/ Institut für Statistik, Vienna, Austria, 1991.

[7] B. Weesakul, "The random walk between a reflecting and an absorbing barrier," The Annals of Mathematical Statistics, vol. 32, no. 3, pp. 765-769, 1961.

[8] G. Lehner, "One dimensional random walk with a partially reflecting barrier," The Annals of Mathematical Statistics, vol. 34, no. 2, pp. 405-412, 1963.

[9] H. Gupta, "Random walk in the presence of a multiple function barrier," Journal of Mathematical Sciences, vol. 1, pp. 18-29, 1966.

[10] S. Dua, S. Khadilkar, and K. Sen, "A modified random walk in the presence of partially reflecting barriers," Journal of Applied Probability, vol. 13, no. 1, pp. 169-175, 1976.

[11] O. E. Percus, "Phase transition in one-dimensional random walk with partially reflecting boundaries," Advances in Applied Probability, vol. 17, no. 3, pp. 594-606, 1985.

[12] M. El-Shehawey, "Absorption probabilities for a random walk between two partially absorbing boundaries: I," Journal of Physics A: Mathematical and General, vol. 34, no. 49, pp. 9005-9013, 2000.

[13] T. Gowers, J. Barrow-Green, and I. Leader, The Princeton Companion to Mathematics, Princeton University Press, Princeton, NJ, USA, 2008.

[14] P. Dirichlet, Abhandlungen der königlich preussischen akademie der wissenschaften, Königlich Preussische Akademie der Wissenschaften zu Berlin, Berlin, Germany, pp. 99-116, 1850, https://www.biodiversitylibrary.org/bibliography/42156. 
[15] T. Kurtz, "A control formulation for constrained markov processes," Mathematics of Random Media, vol. 27, pp. 139150, 1991.

[16] D. L'epingle, Boundary Behavior of a Constrained Brownian Motion between Reflecting-Repellent Walls, University of Wrocław, Wrocław, Poland, 2009, https://www.math.uni. wroc.pl/ pms/files/30.2/Article/30.2.7.pdf.

[17] S. N. Majumdar, J. Randon-Furling, M. J. Kearney, and M. Yor, "On the time to reach maximum for a variety of constrained brownian motions," Journal of Physics A: Mathematical and Theoretical, vol. 41, no. 36, Article ID 365005, 2008.

[18] M. Ormeci, J. G. Dai, and J. V. Vate, "Impulse control of brownian motion: the constrained average cost case," Operations Research, vol. 56, no. 3, pp. 618-629, 2008.

[19] A. Budhiraja and P. Dupuis, "Simple necessary and sufficient conditions for the stability of constrained processes," SIAM Journal on Applied Mathematics, vol. 59, no. 5, pp. 1686-1700, 1999.

[20] A. Budhiraja and P. Dupuis, "Large deviations for the emprirical measures of reflecting brownian motion and related constrained processes in $\$ \mathrm{R}_{-}+\$$," Electronic Journal of Probability, vol. 8, 2003.

[21] I. Kharroubi, J. Ma, H. Pham, and J. Zhang, "Backward sdes with constrained jumps and quasi-variational inequalities," The Annals of Probability, vol. 38, no. 2, pp. 794-840, 2010.

[22] A. Taranto and S. Khan, "Bi-directional grid absorption barrier constrained stochastic processes with applications in finance \& investment," Risk Governance and Control: Financial Markets and Institutions, vol. 10, no. 3, pp. 20-33, 2020a.

[23] A. Taranto and S. Khan, "Drawdown and drawup of bi-directional grid constrained stochastic processes," Journal of Mathematics and Statistics, vol. 16, no. 1, pp. 182-197, 2020b.

[24] A. Taranto and S. Khan, "Gambler's ruin problem and bidirectional grid constrained trading and investment strategies," Investment Management and Financial Innovations, vol. 17 , no. 3, pp. 54-66, 2020c.

[25] A. Taranto and S. Khan, "Application of bi-directional grid constrained stochastic processes to algorithmic trading," Journal of Mathematics and Statistics, vol. 17, no. 1, pp. 22-29, 2021.

[26] C. Zalinescu, Convex Analysis in General Vector Spaces, Singapore, 2002.

[27] H. Bauschke and P. Combettes, Convex Analysis and Monotone Operator Theory in Hilbert Spaces, Springer International Publishing, Berlin, Germany, 2nd edition, 2011.

[28] D. Simpson and R. Kuske, Stochastically Perturbed Sliding Motion in Piecewise-Smooth Systems, 2018, https://arxiv.org/ abs/1204.5792. 